MOLECULAR MOTORS

\section{Moving in a new direction}

Kinesins are molecular motors that drive transport along microtubules, either towards the plus end or towards the minus end, depending on the position of their motor domain. Kinesins with an amino-terminal motor, such as Kinesin- 5 family members, are thought to move towards microtubule plus-ends. However, Roostalu et al. now show that, although the budding yeast Kinesin- 5 chromosome instability 8 (Cin8) is plus-end directed when it is working in a group of motors, single Cin8 motors move towards microtubule minus ends.

The authors first examined the motility of single Cin8 proteins on single immobilized microtubules in vitro using total internal reflection fluorescence microscopy. Surprisingly, these motors moved towards microtubule minus ends in an ATP-dependent manner. Thus, single Cin8 motors move in the opposite direction to that previously thought for wild-type $\mathrm{N}$-terminal kinesins.

Cin8 extends the yeast anaphase spindle by sliding antiparallel microtubules apart through plus-end-directed motion. To see whether Cin8 moves towards the plus end when it is crosslinking two microtubules in vitro, the authors labelled antiparallel microtubule pairs with different fluorescent markers; one microtubule was then immobilized on a surface and the other was crosslinked to green fluorescent protein (GFP)-tagged Cin8. Although crosslinked microtubules were moved by plus-end-directed Cin 8 motility, $\mathrm{Cin} 8$ accumulated at the minus ends of single microtubules. Thus, depending on the circumstances, in 8 can move along microtubules in either direction.

But what exactly determines the direction of Cin 8 motility? The authors performed microtubule-gliding experiments, in which multiple motors that are immobilized on a surface transport microtubules. As immobilized Cin8 motors were plus-end directed, they could move in this direction without the need to crosslink microtubules. However, Cin8 moved towards microtubule minus ends at low protein concentrations, and in both directions at intermediate concentrations. Thus, the number of motors interacting with a microtubule determines the direction of transport, with $\mathrm{Cin} 8$ motors that are working together moving towards microtubule plus ends.

Finally, the authors confirmed their findings in vivo. Yeast Cin8-GFP that was targeted to the cytoplasm, where individual microtubules can be more easily visualized, accrued near microtubule minus ends. Thus, Cin8 is a minus-end-directed motor on individual microtubules in vivo.

So, Cin8 is a bidirectional kinesin that switches the direction of its movement depending on whether it is moving alone or in a group. Future studies are likely to reveal whether other $\mathrm{N}$-terminal kinesins are also bidirectional.

Katharine H. Wrighton

ORIGINAL RESEARCH PAPER Roostalu, J. et al. Directional switching of the kinesin Cin8 through motor coupling. Science 24 Feb 2011 (doi:10.1126/science.1199945) FURTHER READING Veigel, C. \& Schmidt, C. F. Moving into the cell: single-molecule studies of molecular motors in complex environments. Nature Rev. Mol. Cell Biol. 12, 163-176 (2011)| Hirokawa, N. et al. Kinesin superfamily motor proteins and intracellular transport. Nature Rev. Mol. Cell Biol. 10, 682-696 (2009) 